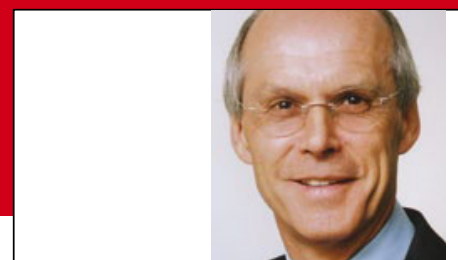

Prof. Dr. med. H. S. FüeßI

Isar-Amper-

Klinikum,

KI. München-Ost,

Haar

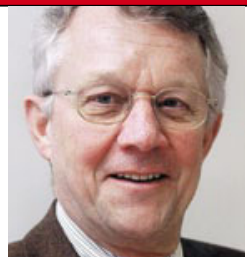

Prof. Dr. med. H. Holzgreve

Internist,

Kardiologische

Praxis, München

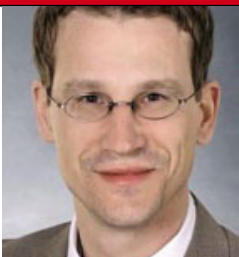

Dr. med. S. Ott

Universitätsklinik für Pneumologie. Inselspital, Bern

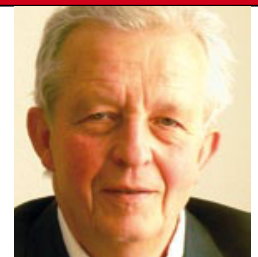

Prof. Dr. med. K. Malberg

Immunologie,

Dresden-

Loschwitz

\title{
Fünf Empfehlungen für eine bessere Medizin
}

\section{Die National Physicians Alliance (NPA) - eine Vereinigung von Allge- meinmedizinern, Internisten und Pä- diatern - hat es sich zum Ziel gesetzt, die Qualität der medizinischen Ver- sorgung zu verbessern. Die Grund- versorgung soll wirtschaftlicher und effizienter werden. Um dieses Ziel zu erreichen, hat die NPA für jede der vertretenen Disziplinen eine Liste der fünf wichtigsten Empfehlungen aufgestellt.}

- Zunächst wurden von NPA-Mitgliedern Vorschläge erarbeitet, die anhand der Literatur auf medizinische Evidenz geprüft wurden. Erste, provisorische Top-5-Listen wurden sodann von 172 NPA-Mitgliedern in der Praxis getestet und noch einmal überarbeitet.
Und so sehen die Empfehlungen aus: Allgemeinmedizin:

(1) bei Rückenschmerzen innerhalb der ersten sechs Wochen möglichst keine Bildgebung;

(2) keine routinemäßige Verschreibung von Antibiotika bei Sinusitiden;

3 kein Routine-EKG bei symptomlosen Patienten mit niedrigem KHK-Risiko;

4 PAP-Tests erst ab 21 Jahren;

5 Knochendichtemessung für Frauen erst ab 65 Jahren und für Männer ab 70 Jahren, außer es sind Risikofaktoren bekannt.

\section{Innere Medizin:}

(1) bei Rückenschmerzen innerhalb der ersten sechs Wochen möglichst keine Bildgebung;

2 keine Routineblut- oder Urintests bei symptomlosen gesunden Erwachsenen;

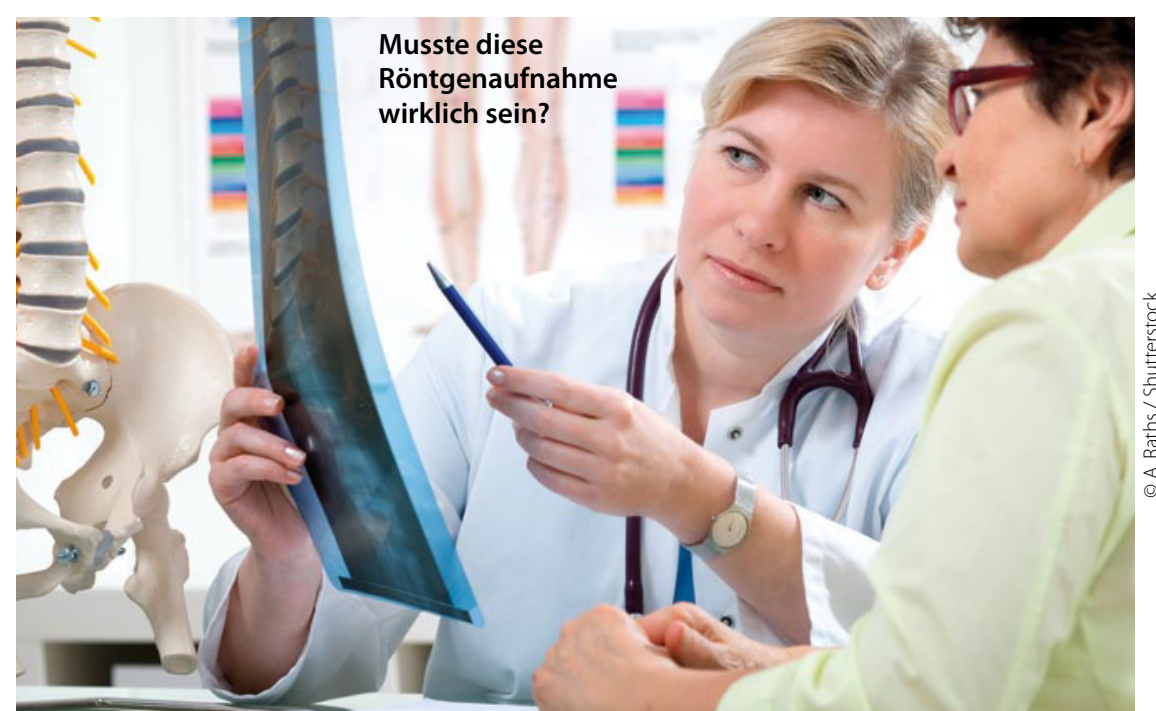

3 kein Routine-EKG bei symptomlosen Patienten mit niedrigem KHK-Risiko;

(4) Einstieg in eine Statintherapie immer mit einem Generikum;

5 Knochendichtemessung für Frauen erst ab 65 Jahren und für Männer ab 70 Jahren, außer es sind Risikofaktoren bekannt.

\section{Pädiatrie:}

(1) Bei Halsschmerzen erst dann Antibiotika verordnen, wenn Streptokokken nachgewiesen sind;

2 keine Bildgebung bei leichteren Kopfverletzungen, wenn der Patient nicht das Bewusstsein verloren hat;

(3) bei Otitis media soll nicht zu früh an den Spezialisten überwiesen werden;

4 Eltern anweisen, ihren Kindern keine rezeptfreien Husten- oder Erkältungsmittel zu geben, da diese unwirksam und möglicherweise mit Nebenwirkungen belastet sind;

(5) bei Asthmakindern sicherstellen, dass der richtige Gebrauch von Cortisonsprays beherrscht wird.

K. MALBERG =

\section{- I. Aguilar et al.}

The „Top 5" lists in primary care: meeting the responsibility of professionalism, the good stewardship working group. Published online May 23, 2011 in Arch Int Med doi: 10.1001/ archinternmed.2011.231 